\title{
WHICH CANONICAL ALGEBRAS ARE DERIVED EQUIVALENT TO INCIDENCE ALGEBRAS OF POSETS?
}

\author{
SEFI LADKANI
}

\begin{abstract}
We give a full description of all the canonical algebras over an algebraically closed field that are derived equivalent to incidence algebras of finite posets. These are the canonical algebras whose number of weights is either 2 or 3 .
\end{abstract}

This note concerns the characterization of the canonical algebras over an algebraically closed field that are derived equivalent to incidence algebras of finite partially ordered sets (posets), expressed in the following theorem.

Theorem. Let $\Lambda$ be a canonical algebra of type $(\mathbf{p}, \boldsymbol{\lambda})$ over an algebraically closed field. Then $\Lambda$ is derived equivalent to an incidence algebra of a poset if and only if the number of weights of $\mathbf{p}$ is either 2 or 3.

This theorem can be interpreted both geometrically and algebraically. From a geometric viewpoint, by considering modules over incidence algebras as sheaves over finite spaces [8] and using the derived equivalence between the categories of modules over a canonical algebra and coherent sheaves over a weighted projective line [3, we are able to obtain explicit derived equivalences between the categories of sheaves of finite dimensional vector spaces over certain finite $T_{0}$ topological spaces and the categories of coherent sheaves over certain weighted projective lines.

From an algebraic viewpoint, in an attempt to classify all piecewise hereditary incidence algebras over an algebraically closed field, one first asks which types of piecewise hereditary categories can actually occur. Happel's classification [6] tells us that we only need to consider the canonical algebras and path algebras of quivers. For the canonical algebras the theorem above gives a complete answer, while for path algebras, see the remarks in Section 2.2.

We finally note that for the constructions of incidence algebras derived equivalent to canonical algebras, the assumption that the base field is algebraically closed can be omitted.

Acknowledgement. I would like to thank Helmut Lenzing for useful discussions related to this paper, and the referee for the helpful comments.

\section{Notations}

The canonical algebras were introduced in [11]. Let $k$ be a field, $\mathbf{p}=$ $\left(p_{1}, \ldots, p_{t}\right)$ be a sequence of $t \geq 2$ positive integers (weights), and $\boldsymbol{\lambda}=$ $\left(\lambda_{3}, \ldots, \lambda_{t}\right)$ be a sequence of pairwise distinct elements of $k \backslash\{0\}$. The canonical algebra of type $(\mathbf{p}, \boldsymbol{\lambda})$, is the algebra $\Lambda(\mathbf{p}, \boldsymbol{\lambda})=k Q / I$ where $Q$ is 
the quiver

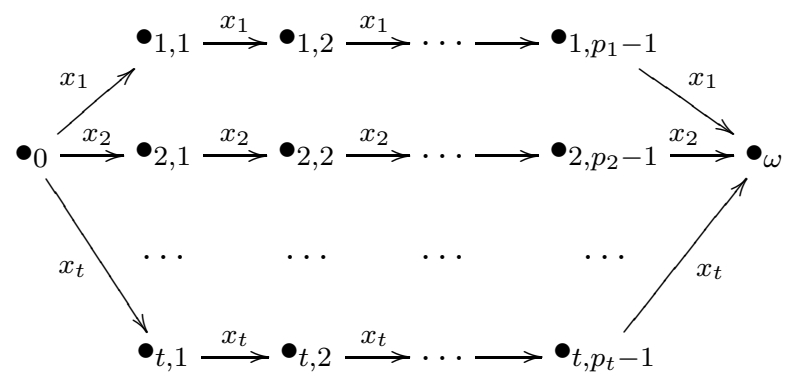

and $I$ is the ideal in the path algebra $k Q$ generated by the following linear combinations of paths from 0 to $\omega$ :

$$
I=\left\langle x_{i}^{p_{i}}-x_{2}^{p_{2}}+\lambda_{i} x_{1}^{p_{1}}: 3 \leq i \leq t\right\rangle
$$

As noted in [11], as long as $t \geq 3$, one can omit weights equal to 1 , and when $t=2$, the ideal $I$ vanishes and the canonical algebra is equal to the path algebra of $Q$. In the latter case, one usually writes only the weights greater than 1, in particular the algebra of type () equals the path algebra of the Kronecker quiver. Hence when speaking on the number of weights, we shall always mean the number of $p_{i}$ with $p_{i} \geq 2$.

Let $X$ be a finite partially ordered set (poset). The incidence algebra $k X$ is the $k$-algebra spanned by the elements $e_{x y}$ for the pairs $x \leq y$ in $X$, with the multiplication defined by setting $e_{x y} e_{y^{\prime} z}=e_{x z}$ if $y=y^{\prime}$ and $e_{x y} e_{y^{\prime} z}=0$ otherwise.

A $k$-diagram $\mathcal{F}$ over $X$ consists of finite dimensional vector spaces $\mathcal{F}(x)$ for $x \in X$, together with linear transformations $r_{x x^{\prime}}: \mathcal{F}(x) \rightarrow \mathcal{F}\left(x^{\prime}\right)$ for all $x \leq x^{\prime}$, satisfying the conditions $r_{x x}=1_{\mathcal{F}(x)}$ and $r_{x x^{\prime \prime}}=r_{x^{\prime} x^{\prime \prime}} r_{x x^{\prime}}$ for all $x \leq x^{\prime} \leq x^{\prime \prime}$. The category of finite dimensional right modules over $k X$ is equivalent to the category of $k$-diagrams over $X$, see 8 .

For a finite-dimensional algebra $\Lambda$ over $k$, we denote by $\mathcal{C}^{b}(\Lambda)$ the category of bounded complexes of (right) finite-dimensional $\Lambda$-modules, and by $\mathcal{D}^{b}(\Lambda)$ the bounded derived category. The algebra $\Lambda$ is piecewise hereditary if $\mathcal{D}^{b}(\Lambda)$ is equivalent as triangulated category to $\mathcal{D}^{b}(\mathcal{H})$ for a hereditary $k$-category $\mathcal{H}$.

\section{The necessity of the Condition $t \leq 3$ in the Theorem}

For a $k$-algebra $\Lambda$, denote by $\mathrm{HH}^{i}(\Lambda)$ the $i$-th Hochschild cohomology of $\Lambda$, which equals $\operatorname{Ext}_{\Lambda \otimes \Lambda^{o p}}^{i}(\Lambda, \Lambda)$, where $\Lambda$ is considered as a $\Lambda$ - $\Lambda$-bimodule in the natural way.

Proposition 1.1. Let $X$ be a poset such that $k X$ is piecewise hereditary. Then $\mathrm{HH}^{i}(k X)=0$ for any $i>1$.

Proof. First, since for any two finite-dimensional $k$-algebras $\Lambda_{1}, \Lambda_{2}$ we have $\operatorname{HH}^{i}\left(\Lambda_{1} \oplus \Lambda_{2}\right)=\operatorname{HH}^{i}\left(\Lambda_{1}\right) \oplus \mathrm{HH}^{i}\left(\Lambda_{2}\right)$, we may assume that $X$ is connected, as the decomposition of $X$ into connected components $X=\sqcup_{i=1}^{r} X_{i}$ induces a decomposition of the incidence algebra $k X=\bigoplus_{i=1}^{r} k X_{i}$.

Let $k_{X}$ be the constant diagram on $X$, defined by $k_{X}(x)=k$ for all $x \in X$ with all maps being the identity on $k$. By [8, Corollary 3.20], $\mathrm{HH}^{i}(k X)=$ $\operatorname{Ext}_{X}^{i}\left(k_{X}, k_{X}\right)$. 


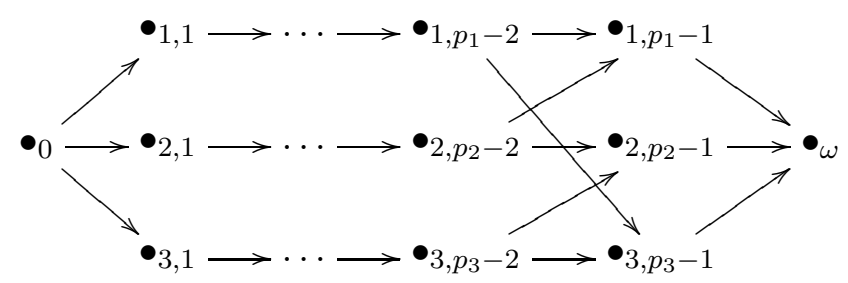

$3 \leq p_{1} \leq p_{2} \leq p_{3}$
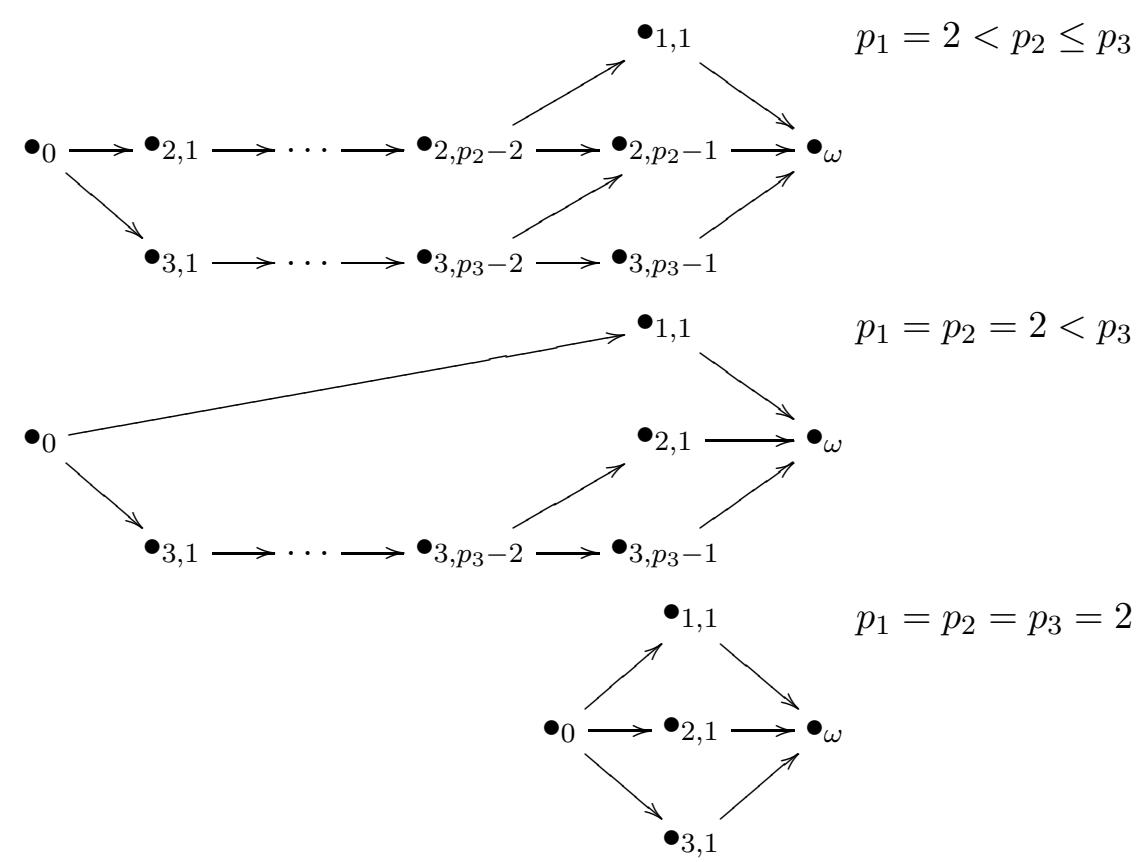

FiguRE 1. Hasse diagrams of posets derived equivalent to canonical algebras of type $\left(p_{1}, p_{2}, p_{3}\right)$.

Since $X$ is connected, $k_{X}$ is indecomposable, and by [4, IV (1.9)], the groups $\operatorname{Ext}_{X}^{i}\left(k_{X}, k_{X}\right)$ vanish for $i>1$, hence $\operatorname{HH}^{i}(k X)=0$ for $i>1$.

Corollary 1.2. Let $k$ be algebraically closed and let $\Lambda$ be a canonical algebra over $k$ of type $(\mathbf{p}, \boldsymbol{\lambda})$ where $\mathbf{p}=\left(p_{1}, \ldots, p_{t}\right)$. If $\mathcal{D}^{b}(\Lambda) \simeq \mathcal{D}^{b}(k X)$ for some poset $X$, then $t \leq 3$.

Proof. Assume that $t \geq 4$. Then $\Lambda$ is not of domestic type and by [5, Theorem 2.4], $\operatorname{dim}_{k} \mathrm{HH}^{2}(k X)=t-3$, a contradiction to Proposition [1.1. Therefore $t \leq 3$.

\section{Constructions of posets from CAnonical Algebras}

2.1. The case $t=3$. Recall that when $t=3$, the canonical algebra $\Lambda(\mathbf{p}, \boldsymbol{\lambda})$ is independent of the parameter $\lambda_{3}$, so we may assume that $\lambda_{3}=1$, and denote the algebra by $\Lambda(\mathbf{p})$. Let $\mathbf{p}=\left(p_{1}, p_{2}, p_{3}\right)$ be a triplet of weights with $2 \leq p_{1} \leq p_{2} \leq p_{3}$. Attach to $\mathbf{p}$ a poset $X_{\mathbf{p}}$ whose Hasse diagram is shown in Figure 1. Explicitly, use the Hasse diagram of (2.1) if $p_{1}>2$, (2.2) if $p_{1}=2$ and $p_{2}>2$, (2.3) if $p_{2}=2$ and $p_{3}>2$ and (2.4) if $p_{3}=2$. 
Theorem 2.1. Let $k$ be a field and let $\mathbf{p}=\left(p_{1}, p_{2}, p_{3}\right)$. Then $\Lambda(\mathbf{p})$ is derived equivalent to the incidence algebra of $X_{\mathbf{p}}$.

Proof. The idea of the proof relies on the notion of a formula introduced in [7], and we refer to that paper for more details. We shall first construct a functor $F: \mathcal{C}^{b}\left(k X_{\mathbf{p}}\right) \rightarrow \mathcal{C}^{b}(\Lambda(\mathbf{p}))$ that induces a triangulated functor $\widetilde{F}: \mathcal{D}^{b}\left(k X_{\mathbf{p}}\right) \rightarrow \mathcal{D}^{b}(\Lambda(\mathbf{p}))$, and then prove that $\widetilde{F}$ is an equivalence.

Note that similarly to the identification in [7] of complexes of diagrams with diagrams of complexes, we may identify complexes of modules over the canonical algebra with a (non-commutative) diagram of complexes of vector spaces satisfying the canonical algebra relations.

For a morphism $f: K \rightarrow L$ of complexes $K=\left(K^{i}, d_{K}^{i}\right), L=\left(L^{i}, d_{L}^{i}\right)$ of vector spaces, denote by $\mathrm{C}(K \stackrel{f}{\rightarrow} L)$ the cone of $f$. Recall that $\mathrm{C}(K \rightarrow$ $L)^{i}=K^{i+1} \oplus L^{i}$, with the differential acting as the matrix

$$
\left(\begin{array}{cc}
-d_{K}^{i+1} & 0 \\
f^{i+1} & d_{L}^{i}
\end{array}\right)
$$

by viewing the terms as column vectors of length two. Denote by [1] the shift operator, that is, $K[1]^{i}=K^{i+1}$ with $d_{K[1]}^{i}=-d_{K}^{i+1}$.

We will demonstrate the construction of $\widetilde{F}$ for the posets of type (2.1), the other cases being similar. Let $K_{0}, K_{\omega}$ and $K_{i, j}$ for $1 \leq i \leq 3,1 \leq j<p_{i}$ be complexes in a commutative diagram

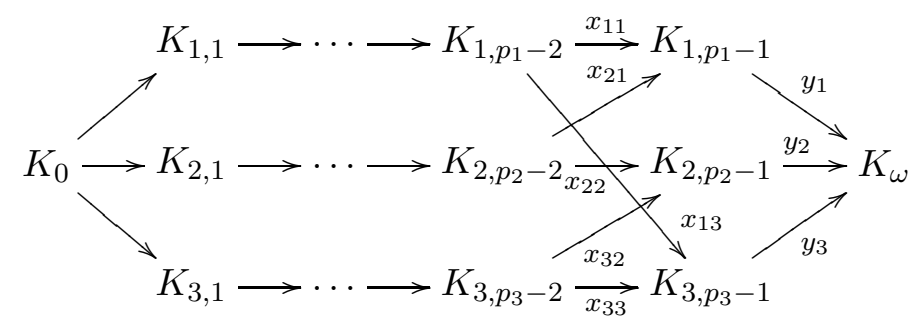

Let $L_{0}=K_{0}$ and $L_{i, j}=K_{i, j}$ for $1 \leq i \leq 3$ and $1 \leq j<p_{i}-1$. Define

$$
\begin{aligned}
L_{1, p_{1}-1} & =\mathrm{C}\left(K_{1, p_{1}-1} \oplus K_{3, p_{3}-1} \stackrel{\left(y_{1} y_{3}\right)}{\longrightarrow} K_{\omega}\right)[-1] \\
L_{2, p_{2}-1} & =\mathrm{C}\left(K_{2, p_{2}-1} \oplus K_{1, p_{1}-1} \stackrel{\left(y_{2} y_{1}\right)}{\longrightarrow} K_{\omega}\right)[-1] \\
L_{3, p_{3}-1} & =\mathrm{C}\left(K_{3, p_{3}-1} \oplus K_{2, p_{2}-1} \stackrel{\left(y_{3} y_{2}\right)}{\longrightarrow} K_{\omega}\right)[-1] \\
L_{\omega} & =\mathrm{C}\left(K_{1, p_{1}-1} \oplus K_{2, p_{2}-1} \oplus K_{3, p_{3}-1} \stackrel{\left(y_{1} y_{2} y_{3}\right)}{\longrightarrow} K_{\omega}\right)[-1]
\end{aligned}
$$

with the three maps

$$
\begin{aligned}
& L_{1, p_{1}-2} \stackrel{\left(x_{11}-x_{13} 0\right)^{T}}{\longrightarrow} L_{1, p_{1}-1} \\
& L_{2, p_{2}-2} \stackrel{\left(-x_{22} x_{21} 0\right)^{T}}{\longrightarrow} L_{2, p_{2}-1} \\
& L_{3, p_{3}-2} \stackrel{\left(x_{33}-x_{32} 0\right)^{T}}{\longrightarrow} L_{3, p_{3}-1}
\end{aligned}
$$

and the three maps from $L_{i, p_{i}-1}$ to $L_{\omega}$ being the canonical embeddings. 
Then the following is a (non-commutative) diagram of complexes

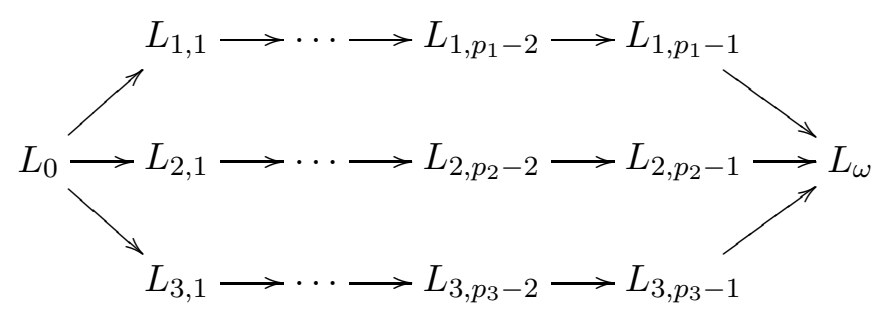

that satisfies the canonical algebra relation, and we get the required functor $F$ which induces, by the general considerations in [7], the functor $\widetilde{F}$.

To prove that $\widetilde{F}$ is an equivalence, we use Beilinson's Lemma [2, Lemma 1] and verify that for any two simple objects $S_{x}, S_{y}$ (where $x, y \in X$ ) and $i \in \mathbb{Z}$, the functor $\widetilde{F}$ satisfies

$$
\operatorname{Hom}_{\mathcal{D}^{b}\left(k X_{\mathbf{p}}\right)}\left(S_{x}, S_{y}[i]\right) \simeq \operatorname{Hom}_{\mathcal{D}^{b}(\Lambda(\mathbf{p}))}\left(\widetilde{F} S_{x}, \widetilde{F} S_{y}[i]\right)
$$

and moreover the images $\widetilde{F} S_{x}$ generate $\mathcal{D}^{b}(\Lambda(\mathbf{p}))$ as a triangulated category.

We omit the details of this verification. However, we just mention that $\widetilde{F} S_{0}$ and $\widetilde{F} S_{i, j}$, for $1 \leq i \leq 3$ and $1 \leq j<p_{i}-1$, are the corresponding simple $\Lambda(\mathbf{p})$-modules, while $\widetilde{F} S_{1, p_{1}-1}, \widetilde{F} S_{2, p_{2}-1}, \widetilde{F} S_{3, p_{3}-1}$ and $\widetilde{F} S_{\omega}$ are given by
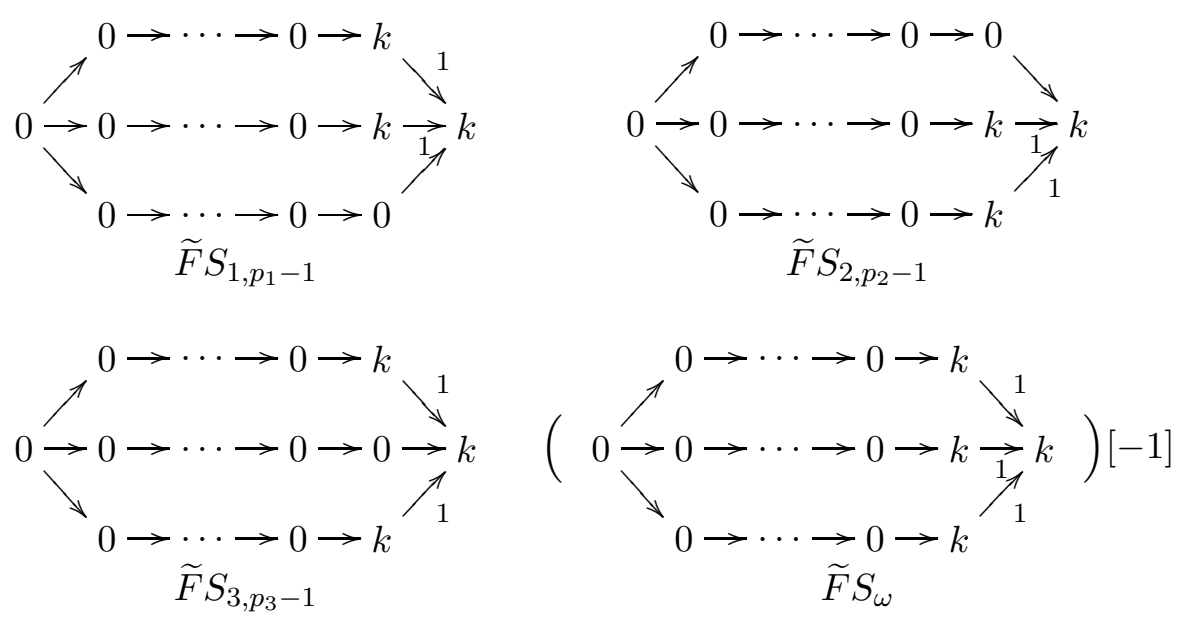

Example 2.2 ([9, Example 18.6.2]). Let $A_{2}$ be the quiver let $X=A_{2} \times A_{2} \times A_{2}$. Then the incidence algebra of $X$ is derived equivalent to the canonical algebra of type $(3,3,3)$.

Remark 2.3. Observe that $\omega$ is the unique maximal element in the posets whose Hasse diagrams are given in (2.3) and (2.4). Hence by taking $Y=\{\omega\}$ in Corollary 4.15 of $[8]$, we recover the fact that the canonical algebra of type $(2,2, p)$ is derived equivalent to the path algebra of the extended Dynkin quiver $\widetilde{D}_{p+2}$.

Remark 2.4. Similar applications of [7, Theorem 1.1] and its corollaries for the posets in (2.2) show that the canonical algebra of type $\left(2, p_{2}, p_{3}\right)$ 
is derived equivalent to the incidence algebras of the posets whose Hasse diagrams are given by

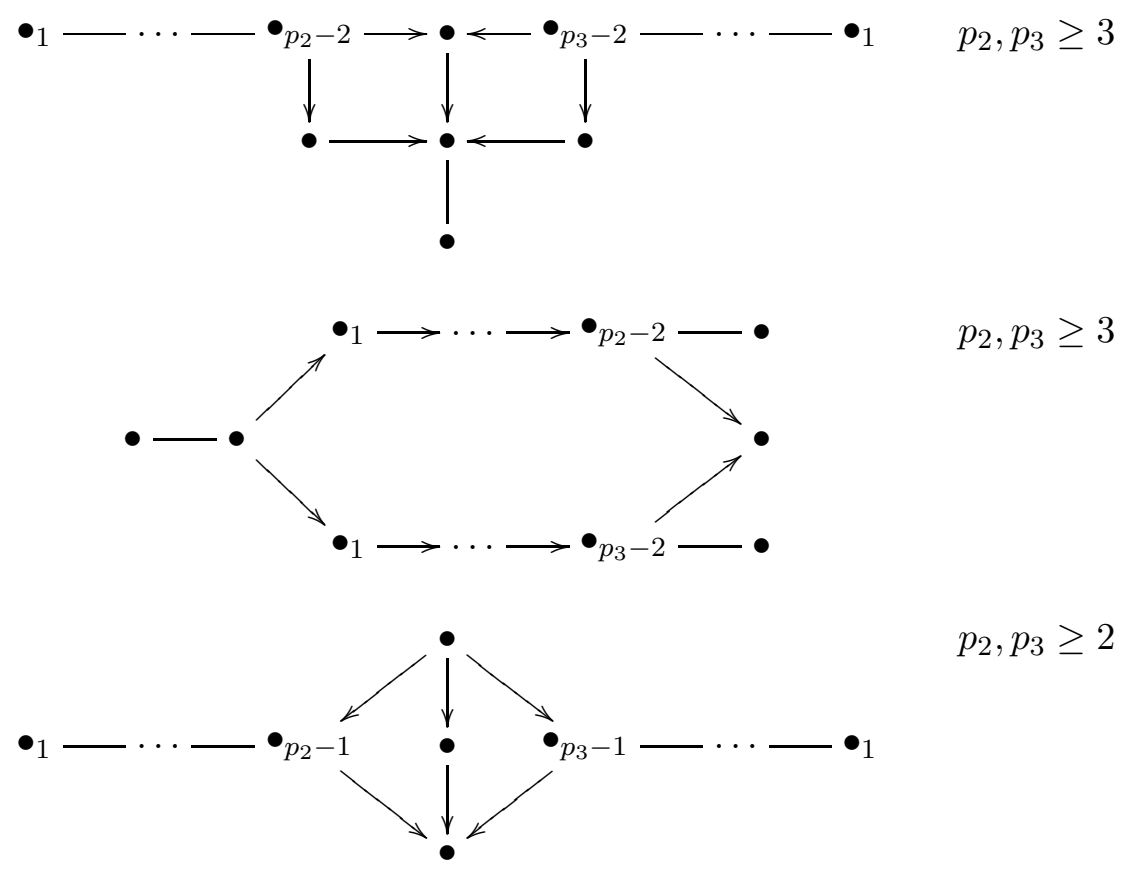

where edges without arrows can be oriented arbitrarily.

2.2. Remarks on path algebras. Let $Q$ be a finite quiver without oriented cycles. The set of its vertices $Q_{0}$ has a natural partial order defined by $x \leq y$ for two vertices $x, y \in Q_{0}$ if there exists an oriented path from $x$ to $y$. When $Q$ has the property that any two vertices are connected by at most one oriented path, the path algebra $k Q$ is isomorphic to the incidence algebra of the poset $\left(Q_{0}, \leq\right)$. A partial converse is given by the following lemma.

Lemma 2.5 ([10, Theorem 4.2]). Let $X$ be a poset. Then gl.dim $k X \leq 1$ if and only if any two points in the Hasse diagram of $X$ are connected by at most one path.

For two quivers $Q$ and $Q^{\prime}$, we denote $Q \sim Q^{\prime}$ if $Q^{\prime}$ can be obtained from $Q$ by applying a sequence of BGP reflections (at sources or sinks), see 4, (I.5.7)]. Since BGP reflections preserve the derived equivalence class, we conclude that if $Q$ is a quiver such that $Q \sim Q^{\prime}$ for a quiver $Q^{\prime}$ having the property that any two vertices are connected by at most one oriented path, then the path algebra $k Q$ is derived equivalent to an incidence algebra of a poset.

2.3. The case $t=2$. When the number of weights is at most 2 , the corresponding canonical algebra is a path algebra of a quiver, however there are two distinct paths from the source 0 to the sink $\omega$.

When the weight type is $\left(p_{1}, p_{2}\right)$ (with $p_{1}, p_{2} \geq 2$ ), we can overcome this problem by applying a BGP reflection at the sink $\omega$. The resulting quiver 


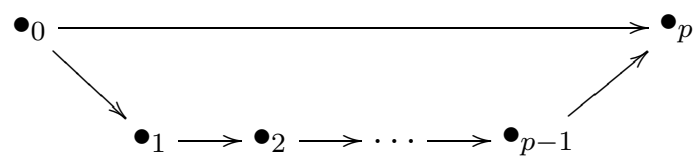

FIgURE 2. The quiver $\widetilde{A}_{1, p}-$ not derived equivalent to any incidence algebra.

is shown below,

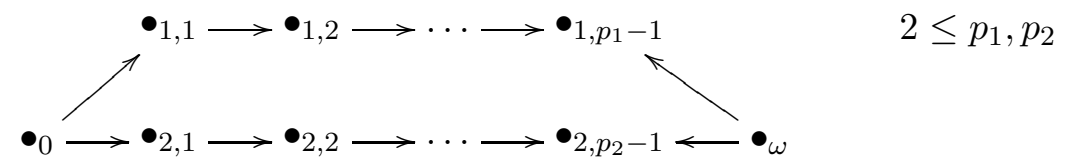

and its path algebra is an incidence algebra derived equivalent to the canonical algebra of type $\left(p_{1}, p_{2}\right)$.

In the remaining case, where the weight type is either $(p)$ or () , the corresponding canonical algebra equals the path algebra of the quiver $\widetilde{A}_{1, p}$ drawn in Figure 2, and we show the following.

Proposition 2.6. Let $p \geq 1$ and let $k$ be algebraically closed. Then there is no poset whose incidence algebra is derived equivalent to the path algebra of the quiver $\widetilde{A}_{1, p}$.

Proof. Assume that there exists a poset $X$ such that $k X$ is derived equivalent to the path algebra of $\widetilde{A}_{1, p}$.

If gl.dim $k X \leq 1$, then by Lemma 2.5, the algebra $k X$ equals the path algebra of its Hasse diagram $Q$, thus by [4, (I.5.7)], $Q$ is obtained from $\widetilde{A}_{1, p}$ by a sequence of BGP reflections. But this is impossible since the only possible reflections are at 0 and $p$, and they give quivers isomorphic to $\widetilde{A}_{1, p}$. However, $\widetilde{A}_{1, p}$ is not the Hasse diagram of any poset.

Hence gl.dim $k X \geq 2$. By Lemma 2.5, there exists at least one commutativity relation in the quiver $Q$, so that $k X$ is not a gentle algebra. This is again impossible since the path algebra of $\widetilde{A}_{1, p}$ is gentle and the property of an algebra being gentle is invariant under derived equivalence [12. Alternatively, one can use the explicit characterization in [1] of iterated tilted algebras of type $\tilde{A}_{n}$.

\section{REFERENCES}

[1] Assem, I., And Skowroński, A. Iterated tilted algebras of type $\tilde{\mathbf{A}}_{n}$. Math. Z. 195, 2 (1987), 269-290.

[2] BeĬlinson, A. A. Coherent sheaves on $\mathbf{P}^{n}$ and problems in linear algebra. Funktsional. Anal. i Prilozhen. 12, 3 (1978), 68-69.

[3] Geigle, W., And Lenzing, H. A class of weighted projective curves arising in representation theory of finite-dimensional algebras. In Singularities, representation of algebras, and vector bundles (Lambrecht, 1985), vol. 1273 of Lecture Notes in Math. Springer, Berlin, 1987, pp. 265-297.

[4] HAPPEL, D. Triangulated categories in the representation theory of finite-dimensional algebras, vol. 119 of London Mathematical Society Lecture Note Series. Cambridge University Press, Cambridge, 1988. 
[5] Happel, D. Hochschild cohomology of piecewise hereditary algebras. Colloq. Math. 78, 2 (1998), 261-266.

[6] HAppel, D. A characterization of hereditary categories with tilting object. Invent. Math. 144, 2 (2001), 381-398.

[7] LADKAnI, S. Universal derived equivalences of posets. arXiv:0705.0946.

[8] Ladkani, S. On derived equivalences of categories of sheaves over finite posets. $J$. Pure Appl. Algebra 212, 2 (2008), 435-451.

[9] Lenzing, H. Coxeter transformations associated with finite-dimensional algebras. In Computational methods for representations of groups and algebras (Essen, 1997), vol. 173 of Progr. Math. Birkhäuser, Basel, 1999, pp. 287-308.

[10] Mitchell, B. On the dimension of objects and categories. II. Finite ordered sets. J. Algebra 9 (1968), 341-368.

[11] Ringel, C. M. Tame algebras and integral quadratic forms, vol. 1099 of Lecture Notes in Mathematics. Springer-Verlag, Berlin, 1984.

[12] Schröer, J., ANd Zimmermann, A. Stable endomorphism algebras of modules over special biserial algebras. Math. Z. 244, 3 (2003), 515-530.

Einstein Institute of Mathematics, The Hebrew University of Jerusalem, JERUSALEM 91904, ISRAEL

E-mail address: sefil@math.huji.ac.il 